Revue d'histoire de l'enfance « irrégulière »

Le Temps de l'histoire

$17 \mid 2015$

Naissance et mutation de la justice des mineurs

\title{
La création de la justice des mineurs au Portugal
}

The creation of children's courts in Portugal

João Fatela

(2) OpenEdition

Journals

Édition électronique

URL : http://journals.openedition.org/rhei/3791

DOI : 10.4000/rhei.3791

ISSN : $1777-540 \mathrm{X}$

Éditeur

Presses universitaires de Rennes

Édition imprimée

Date de publication : 30 octobre 2015

Pagination : $37-64$

ISBN : 978-2-7535-4215-0

ISSN : $1287-2431$

Référence électronique

João Fatela, «La création de la justice des mineurs au Portugal », Revue d'histoire de l'enfance " irrégulière » [En ligne], 17 | 2015, mis en ligne le 30 octobre 2017, consulté le 04 mai 2019. URL : http:// journals.openedition.org/rhei/3791; DOI : 10.4000/rhei.3791 


\section{La création de la justice des mineurs au Portugal}

La création des tribunaux pour enfants au Portugal intervient très tôt dans l'histoire de cette juridiction en Europe, puisqu'elle date de 1911. Mais la définition de leur cadre juridique ne cessera d'évoluer jusqu'aux années 1930 approximativement. Ce sont les bases et les principales étapes de l'établissement de cette juridiction que nous chercherons à décrire ici. De nature essentiellement tutélaire, elle connaît en 1925 un tournant marqué par la pensée de défense sociale. Parallèlement à sa complète dépénalisation, l'approche de la délinquance infantile à partir de cette date, dans un sens plus subjectif, qui va jusqu'à intégrer la notion de prédélinquance, témoigne, de l'influence croissante de la psychologie et de la psychiatrie.

The creation of children's courts in Portugal happened quite early in the history of such jurisdiction in Europe, since it goes back to 1911. But the definition of their legal framework will endlessly evolve until the thirties. What I'll try to describe here are the foundations and main stages of such jurisdiction. Being of a nature mostly tutelary, it took however a dramatic turn in 1925, marked by thoughts of social protection. In parallel to its complete decriminalization, the approach of child delinquency from that year onwards, will testify, in a more subjective sense that will even integrate the notion of pre-delinquency, to the growing influence of psychology and psychiatry.

Mots-clefs : justice des mineurs, Portugal, dépénalisation, éducation, observation, pluridisciplinarité, positivisme, prédélinquance, science de l'enfant, tutélaire, vagabondage

Keywords : juvenile justice, Portugal, discriminalisation, education, observation, pluridisciplinary, positivism, pre-delinquency, science of child, tutelary, vagrancy

L a création des tribunaux pour enfants au Portugal date de $1911^{1}$. Elle interdvient donc très tôt dans l'histoire de cette juridiction en Europe ${ }^{2}$. Le fait contraste avec la politique tardive et erratique menée jusqu'alors par ce pays dans le champ de l'enfance dite "irrégulière ». À l'exception de quelques expériences ponctuelles, le premier établissement pour enfants de justice, la maison

\section{João FATELA}

Docteur en anthropologie et psychologue.

1. Je remercie Eliana Gersão, membre du Centre de droit de la famille de l'université de Coimbra, pour son aide inestimable dans le déchiffrage des subtilités du droit pénal des mineurs au Portugal. Mais les erreurs que ce travail pourrait comporter sont de mon entière responsabilité E. Gersão est l'auteure de nombreuses études sur le sujet et son nom reste lié à deux réformes sur les mineurs de justice réalisées après la révolution démocratique de 1974. La création de la justice des mineurs au Portugal ou plutôt le mouvement éducatif qui la précède et l'accompagne a connu ces dernières années un regain d'intérêt, surtout dans le champ des sciences de l'éducation. Malheureusement ce n'est pas le cas de l'histoire, où les travaux sont pratiquement inexistants, même si parmi ceux effectués dans d'autres disciplines, certains se distinguent par leur dimension historique. C'est le cas notamment de celui de J. M. Santo cité plus loin et de la recherche en partie inédite de M. R. Tomé sur le tribunal central pour enfants de Coimbra. Voir, de 
ce dernier auteur, A Criança e a delinquência juvenil na Primeira Republica, Lisbonne, CPIHTS, 2003 Sans parler de l'excellent ouvrage collectif coordonné par Vieira, Alçada et Barreiros sur les établissements de justice pour mineurs cité plus loin également. II n'empêche, que tout travail historiographique bute actuellement sur la question des sources primaires qui restent en grande partie à localiser, identifier et rassembler. Par ailleurs, on ne peut que regretter que les historiens de la Première République ignorent quasi systématiquement ce qui reste de l'une des plus grandes réformes de cette période historique.

2. Voir DOUPONT-BOUCHAT Sylvie et Pierre Éric, Enfance et justice au XIX siècle, Paris, PUF, 2001

3. Au moment de la création de la justice des mineurs, ce réseau était constitué de trois maisons de correction, dont l'une pour filles, et une colonie correctionnelle agricole.

4. Droux Joëlle, "L'internationalisation de la protection de l'enfance: acteurs, concurrents et projets transnationaux (1900-1925) ", Critique Internationale, juilletseptembre, 2011, p. 19.

5. Oliveira Padre Antonio de, Deixemos os pais cuidemos dos filhos, Lisbonne, 1923, p. LIX. de détention et de correction de Lisbonne (MDCL), réservée aux garçons, n’est en effet créée qu'en 1871. Et son fonctionnement est encore très marqué par le modèle carcéral. Faute d'établissements spécialisés, les mineurs sont relégués pendant de longues années, dans des conditions déplorables, dans les prisons pour adultes. La seule « alternative " à l'incarcération étant pour certains l'armée ou la marine. Remarquons que le recours, de façon volontaire ou compulsive, à l'incorporation militaire ne s'arrête pas avec la création de la justice des mineurs. Celle-ci continue d'y recourir en tant que moyen punitif et disciplinaire ou comme une forme de patronage pour suppléer les difficultés de placement à la sortie d'un établissement. C'est la nouvelle juridiction qui en revanche met un terme à la présence des mineurs dans les prisons communes (il n'y a jamais eu de prisons pour enfants au Portugal) qui s'est en fait poursuivie malgré l'ouverture des premiers établissements de correction. C'est finalement à travers la lente constitution du premier réseau d'établissements de justice pour mineurs ${ }^{3}$ que les idées de protection et d'éducation vont émerger dans le champ pénal, surtout à partir du début du $\mathrm{xx}^{\mathrm{e}}$ siècle. Car le Portugal ne dispose pas avant 1911 de lois "protectionnelles ", comme celle de 1889, en France, sur les enfants victimes de violences physiques ou morales au sein de leurs familles ${ }^{4}$. La publication du règlement de la MDCL, en septembre 1901, marque, malgré ses incohérences, un tournant majeur visant à privilégier l'éducatif sur le punitif. Une orientation qui témoignerait, selon son préambule, de l'intérêt que l'État et la société portent aux mineurs afin que les mesures de correction ne soient pas vécues comme un châtiment. C'est sur la base de ce règlement, dont il est l'auteur, que le sous-directeur de la MDCL, le père Antonio de Oliveira, à qui le gouvernement de la République confiera dix ans plus tard la mise en place de la justice des mineurs, s'engage dans une phase d'importants changements, à travers l'introduction de méthodes pédagogiques innovantes pour le Portugal de l'époque (travaux manuels, jardins scolaires, leçons de choses, gymnastique, etc.), selon le principe: "Éduquer [...] c'est développer ce que chacun a de différent et de supérieur ${ }^{5}$. "Son action s'articule étroitement avec les courants pédagogiques qui se développent à partir $\mathrm{du} \mathrm{XIX}^{\mathrm{e}}$ siècle, en contribuant ainsi à la formation d'une science de l'enfant. Cette phase coïncide avec le transfert, en 1903, de son établissement à Caxias, dans la périphérie de Lisbonne. Car, malgré les problèmes que pose l'adaptation de l'édifice (un ancien couvent, comme auparavant, mais en bord de mer), ce déplacement est pour lui une 
condition indispensable pour rompre avec le modèle carcéral de l'ancienne MDCL, afin d'en faire une école de réforme et d'amener les mineurs à rompre, eux aussi, avec "l'idée qu'ils ont été, sont ou pourraient redevenir criminels ${ }^{6}$ ».

Nous ne savons pas si l'idée d'une juridiction pour mineurs s'est déjà imposée à lui à ce moment-là, mais l'idée circulait au Portugal depuis la fin du $\mathrm{XIX}^{\mathrm{e}}$ siècle. On présente habituellement le VII ${ }^{\mathrm{e}}$ Congrès de l'Union internationale de droit pénal (UIDP), qui a lieu à Lisbonne, en avril 1897, comme le moment où la question d'une "magistrature exclusivement occupée de l'enfance ", selon les termes de la Revue Pénitentiaire, fait l'objet pour la première fois d'un débat public au Portugal' ${ }^{7}$. À l'initiative de l'Association portugaise d'avocats et du groupe portugais de l'UIDP, une séance est consacrée " à la procédure à suivre contre le délinquant mineur tombant sous le coup de la loi pénale ». Du côté portugais, une " magistrature spéciale " pour mineurs est défendue par Ernesto Leite de Vasconcelos, directeur de l'École agricole (future colonie correctionnelle) de Vila Fernando et membre de l'UIDP, qui reprend l'idée, en 1906, lors du XV Congrès international de médecine, qui s'est tenu également dans la capitale portugaise ${ }^{8}$. Ces tentatives n'ont pas de suite. Enfin, en 1909, c'est à l'un des derniers ministres de la justice de la Monarchie, Francisco José de Medeiros, de s'interroger sur l'intérêt des tribunaux spéciaux pour mineurs délinquants, dans l'exposé des motifs d'un projet de loi (avorté) sur les établissements de correction, à contre-courant d'ailleurs du tournant éducatif de 1901. Mais c'est pour aussitôt rejeter l'idée, sous un prétexte manifestement fallacieux. Ce qui manque à l'évidence c'est la volonté politique et ce d'autant que l'initiative privée, dont on sait combien la mobilisation en faveur de la justice des mineurs fut déterminante dans un certain nombre de pays', est tout à fait absente du champ de l'enfance délinquante. Rappelons à ce propos que, à quelques rares exceptions, comme les Oficinas de $S$. José, à Porto, dans la phase initiale, les établissements pour enfants de justice au Portugal sont tous publics jusqu'en 1943.

Il faudra attendre l'instauration de la République, le 5 octobre 1910, pour que le cap soit franchi. Moins de deux mois après cet événement, le ministre de la Justice du gouvernement provisoire, Afonso Costa, le dirigeant républicain le plus influent de l'époque ${ }^{10}$, effectue une visite à la MDCL, qui lui laisse une forte impression. Il demande à revoir le père Antonio de Oliveira, quelques jours plus tard, afin de solliciter sa collaboration pour l'œuvre que la

\author{
6. Oliveira Padre Antonio de, \\ Deixemos..., ibid., p.VI-X. \\ 7. Voir à ce propos la \\ Revue Pénitentiaire, juin et \\ décembre 1897. Parmi les \\ fondateurs de I'UIDP, dont \\ on connaît le rôle en faveur \\ de la protection de l'enfance \\ par le biais de la pensée \\ de défense sociale, ont été \\ présents van Hammel et \\ von Liszt, mais pas Prins. \\ Annoncé, Jules Lejeune \\ s'est finalement fait excuser. \\ 8. VASCONCELOS Ernesto Leite \\ de, «La nécessité de la \\ réforme du système pénal \\ du point de vue de l'obser- \\ vation psychiatrique pour \\ les mineurs délinquants ", \\ Actes du XV congrès \\ international de médecine, \\ section VI, Lisbonne, 1916, \\ p. 578-581. \\ 9. Doupont-Bouchat Sylvie, \\ Enfance et..., op. cit., p. 361 \\ sq.
}

10. Afonso Costa est l'auteur d'un ouvrage assez fouillé sur la criminologie moderne et les perspectives ouvertes par le positivisme dans le droit pénal: Comentario Antonio de Olivera Codigo penal português, Coimbra, Imprensa da Universidade, 1895. S'inscrivant dans la tradition positiviste, il se disait proche de Tarde qui privilégiait les facteurs sociaux dans l'étude de la criminalité. 
11. Oliveira Padre Antonio de, Deixemos os..., op. cit., p. XLVI-VII.

12. « Former l'homme nouveau... Utopie et pédagogie pendant la Révolution Française ", Libre, 8, Paris, 1980, p. 89-132.

13. NovoA Antonio, «A Republica e a escola. Das intenções generosas Antonio de Olivera desengano das realidades ", Revista Portuguesa de Educação, 3 , 1988, p. 29-60.
République souhaite mettre en place en faveur de la protection de l'enfance ${ }^{11}$. Son prestige, en raison de l'action menée dans l'établissement de Caxias, et qui ne s'arrête pas aux frontières du Portugal, fait de lui la personne indiquée. Mais dans le contexte d'anticléricalisme exacerbé qui alors fait rage, orchestré par Afonso Costa lui-même, la nomination d'un prêtre catholique pour une mission si emblématique pour les républicains a de quoi surprendre. Sans doute, Afonso Costa a compris le bénéfice politique qu'il peut tirer de cette nomination. En atténuant l'impact de son intolérance envers l'Église catholique aux yeux de l'opinion, celle-ci contribue du coup à légitimer le nouveau régime. Parallèlement, dans un geste irréfutable de réparation matérielle et symbolique, la loi de séparation des Églises et de l'État stipulait que les biens de l'Église ou appartenant aux congrégations religieuses expulsées ou dissoutes suite à l'instauration de la République soient en partie affectés à « l'œuvre de préservation des mineurs en danger moral » que la création des tribunaux pour enfants allait bientôt concrétiser. De son côté, le père Antonio de Oliveira fait part, dans un entretien au journal $O$ Século, du 11 février 1911, de son espoir de voir la République entreprendre à une plus large échelle ce qu'il a engagé au sein de la MDCL: «soumettre tous les établissements pour enfants aux principes modernes de la pédagogie, en mettant en œuvre une éducation intégrale qui assure le développement harmonieux de la dimension physique et de la dimension morale... » Ce qui, c'est le cas de dire, valait bien une messe... Connaissant l'intérêt du nouveau régime pour l'éducation, il tente de saisir l'opportunité qui lui est offerte pour faire avancer ses idées, sans se préoccuper du fait que, pour les dirigeants républicains, la question constitue un enjeu crucial dans leur combat pour la laïcité. Antonio Novoa, s'appuyant sur les travaux de Bronislaw Baczo $^{12}$ sur les rapports entre utopie et pédagogie sous la Révolution française, montre combien l'éducation a avant tout pour les républicains une dimension utopique, car la "société nouvelle " qu'ils visaient ne pouvait avoir lieu sans « un homme nouveau, formé au sein d'institutions affranchies de l'esprit jésuitique et monarchique ${ }^{13}$ ». Fernando Catroga insiste également sur ce point, lorsqu'il évoque le rôle du positivisme dans la consolidation de l'idéologie républicaine: " (les positivistes) se battaient pour la laïcisation totale des consciences et la sécularisation des institutions en vue de l'établissement d'une mentalité libérée définitivement de l'aliénation religieuse ". Au-delà de l'idéologie républicaine, l'importance du positivisme au Portugal transparaît dans des domaines du savoir 
aussi divers que l'anthropologie, la biologie, la médicine, la psychiatrie, la pédagogie ou le droit ${ }^{14}$. La justice des mineurs en porte la trace, comme nous le verrons. Mais sur le plan politique, c'est indéniablement la sensibilité, l'intérêt des républicains pour l'éducation, indépendamment des résultats plutôt mitigés de leur action dans le champ scolaire, qui a favorisé cette réforme. Une réforme que le père Antonio de Oliveira inscrit, lui aussi, dans le registre de l'utopie, en écrivant dans l'exposé des motifs de la loi fondatrice de la justice des mineurs, que celle-ci incarne "le rêve patriotique de régénération de la famille portugaise », voire d'une "nationalité nouvelle », puisqu'elle veut éduquer, purifier et améliorer l'enfant.

La mission confiée par Afonso Costa au père Antonio de Oliveira a débouché, dans un premier temps, sur la création à Lisbonne le $1^{\text {er }}$ janvier 1911 d'une Commission de protection des mineurs en danger moral, pervertis et délinquants dans un but de préservation et de réforme. Une commission du même type aurait été créée à Porto le 4 février suivant sur laquelle nous ne disposons d'aucune information. Circonscrite à la capitale, la commission de Lisbonne est présidée par le préfet, mais son vrai animateur est bien sûr le père Antonio de Oliveira, promu par la même occasion surintendant des trois maisons de détention et de correction alors existantes. Sans pouvoir juridictionnel, les attributions de cette commission sont, comme le nom l'indique, d'ordre tutélaire. Elle est chargée de formuler dans les plus brefs délais un plan national de prévention et de réforme de la jeunesse portugaise en danger moral, pervertie et délinquante, tout en apportant une réponse immédiate, sur la base d'une enquête préalable, à la situation dans la capitale, où l'existence d'un grand nombre d'enfants vivant dans des " conditions d'extrême abandon ou livrés à une dégradante immoralité et perversion ", exigeait des mesures urgentes. Il s'agit plus concrètement de mineurs de moins de 16 ans trouvés sur la voie publique sans abris ni moyens de subsistance ou se livrant au vagabondage, à la mendicité ou au libertinage. Dès février, la commission ouvre un espace appelé " dépôt ", où sont détenus provisoirement et examinés les mineurs, en attendant qu'une solution soit trouvée. Les informations dont nous disposons sur l'activité de cette commission sont éparses et peu nombreuses. Au-delà de l'accueil, de l'examen et de la réorientation des mineurs, elle serait à l'origine de quelques initiatives pédagogiques innovantes bien qu'infructueuses ${ }^{15}$. Elle

\author{
14. Catroga Fernando, «A \\ influência do positivismo na \\ consolidação da ideologia \\ republicana em Portugal ", \\ Biblos, vol. LIII, 1977, \\ p. 285-327. Sur les liens \\ entre le positivisme, notam- \\ ment dans ses rapports \\ avec l'anticléricalisme, et la \\ libre-pensée, dont l'influence \\ chez les républicains fut \\ également déterminante, \\ voir, du même auteur, " $\mathrm{O}$ \\ livre-pensamento contra a \\ Igreja ", Revista de historia \\ das ideias, vol. 22, 2001, \\ p. 255-354. \\ 15. Barreto Lima, Padre \\ Antonio de Oliveira, Caxias, \\ s. d., p. 58-59.
}


16. Castro Pedro, A Tutoria, janvier 1913, p. 57

17. OLiveira Padre Antonio de, Deixemos os..., op. cit., p. LXI-LXIII.

18. Oliveira Padre Antonio de, Via infamante, Lisbonne, s. d., p. 133-135 joue par ailleurs un rôle précurseur dans l'émergence de la justice des mineurs qu'elle préfigure à plusieurs titres.

C'est à partir des travaux de cette commission, que le père Antonio de Oliveira va rédiger le décret-loi du 27 mai 1911, connu également sous le nom de loi de protection de l'enfance (LPE), qui établit la justice des mineurs ${ }^{16}$, avec ses trois grands volets institutionnels: les tribunaux pour enfants; les refuges annexes à certains tribunaux destinés à la garde temporaire et à l'observation des mineurs et la Fédération nationale des amis et défenseurs des enfants, définie comme "l'union juridique, morale et facultative de diverses institutions officielles ou privées de propagande, éducation et patronage, qui devront former un vrai système d'hygiène morale et sociale. " Cette fédération devient, en 1928, sous la désignation de Fédération nationale des institutions de protection de l'enfance, la section portugaise de l'Association internationale de la protection de l'enfance (AIPE). Nous ne pourrons nous consacrer malheureusement qu'aux deux premiers volets. Il serait intéressant de connaître les circonstances exactes dans lesquelles la nouvelle juridiction est amenée à succéder à la commission de protection ainsi que la teneur des débats auxquels cette décision a donné lieu. Le père Antonio de Oliveira observe, en effet, que le texte publié au journal officiel n'est qu'un «brouillon » de ce qu'il avait préparé, la loi ayant été « mutilée de ses dispositions les plus intéressantes ${ }^{17}$ ". Malgré un contexte politique favorable, la décision de créer une telle juridiction n'allait pas de soi, y compris en ce qui concerne le père Antonio de Oliveira. Si celui-ci a toujours dénoncé avec force la stigmatisation liée au passage des mineurs par les tribunaux ordinaires et les prisons, nous ne lui connaissons pas de position formelle en sa faveur avant 1911. Sauf peut-être dans son ouvrage, Via Infamante, où elle apparaît en creux à travers l'appel à la fermeture de ces institutions aux mineurs de moins de 16 ans qui clôt le dernier chapitre. Celui-ci est consacré presque exclusivement à l'intervention du ministre belge de la Justice, Jules Lejeune, le 20 novembre 1891, au Sénat, dans laquelle il dénonce « l'engrenage judiciaire » qui arrête et étiquette, de façon indiscriminée, des milliers d'enfants ${ }^{18}$. Par son style même, la LPE témoigne de l'urgence dans laquelle elle est rédigée. En raison des élections pour l'Assemblée constituante qui vont se dérouler dès le lendemain de sa promulgation, il est important qu'elle soit finalisée rapidement pour que les nouveaux députés puissent s'en saisir. C'est d'ailleurs l'une des raisons évoquées dans l'exposé des motifs pour expliquer son caractère expérimental: 
l'application de la LPE est circonscrite dans un premier temps au tribunal central pour enfants de Lisbonne et ne s'adresse qu'aux enfants maltraités, délaissés et délinquants. La suite dépendrait des premiers résultats. Mais tout indique que l'Assemblée constituante n'en a jamais été saisie.

Si la LPE constitue l'acte fondateur de la justice des mineurs, celle-ci ne gagne ses véritables contours qu'au cours d'un processus juridique laborieux et tâtonnant, fait de réajustements successifs, qui se prolonge jusqu'aux années 1930 approximativement. Il correspond à ce que Bandeira appelle "la phase expérimentale de construction " de la justice des mineurs ${ }^{19}$. Une étape marquante de ce processus est la promulgation du décret $10767 \mathrm{du}$ 15 mai 1925, suivie d'un ensemble de mesures législatives et administratives qui complètent et redéfinissent certaines de ses dispositions. Mais le modèle instauré en 1911 ne connaît de changements significatifs qu'en 1962, avec la promulgation de l'Organisation tutélaire de mineurs. Entre 1930 et 1950, l'État lance un vaste programme de construction d'établissements judiciaires pour mineurs (ad hoc) d'après le plan défini vers $1927^{20}$. Quant à la LPE, elle ne perdra jamais sa dimension référentielle, comme le souligne Eliana Gersão, en 1984:

toutes les mesures vraiment importantes et décisives, dans le cadre de l'inter-

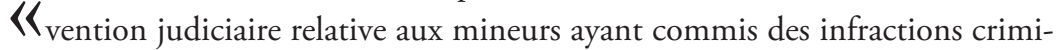
nelles, ont été introduites [...] par la LPE [...] Ce sont ses principes et son idéologie - formulés de différentes manières et poussés parfois jusqu’à l'extrême - qui ont marqué toute l'évolution de notre législation tutélaire et dominent encore aujourd'hui le système en vigueur ${ }^{21}$ ".

On remarquera par ailleurs l'étonnant consensus dont la réforme fait l'objet au long des régimes politiques si contrastés qui couvrent la période correspondant à sa mise en place: la Première République parlementaire, instaurée en 1910 et qui se termine en 1926; la dictature autoritaire de Salazar, qui débute formellement en 1933 et s'étire pendant quatre décennies, avec, entre les deux régimes, une dictature militaire un peu singulière qui met un terme à la République et ouvre la route à Salazar. Même si chaque régime n’hésitera pas à la décliner selon ses propres présupposés idéologiques.

D'après la LPE, le tribunal pour enfants, qu'elle désigne pour tutoria da infância, est « un tribunal collectif spécial, essentiellement équitable, qui a pour

\author{
19. BANDEIRA Filomena, \\ "A formação da rede \\ nacional de estabelecimen- \\ tos judiciais de internamento \\ para menores ", VIEIRA João, \\ Alcada Margarida, BarReIros \\ Maria Helena, Arquitectura \\ dos serviços publicos em \\ Portugal. Os internatos na \\ justiça dos menores 1871 - \\ 1978, Lisbonne, Ministério \\ de Justiça et Instituto de \\ Habitação e da Reabilitação \\ Urbana, 2009, p. 65 sq. \\ 20. Bandeira Filomena, \\ « A formação... ", ibid., \\ p. 37 sq. \\ 21. Gersao Eliana, \\ «Menores agentes de \\ informações criminais. Que \\ intervenção? ", Estudos de \\ homenagem ao Prof. Doutor \\ Eduardo Correia, vol. I, \\ numéro spécial du Boletim \\ da Faculdade de Direito da \\ Universidade de Coimbra, \\ Coimbra, 1984, p. 623.
}


22. CAstro Pedro, Rapport présenté au Congrès international de protection de l'enfance (23-26 juillet 1913), Lisbonne, 1913, p. 8. but de préserver, défendre et protéger les mineurs en danger moral, délaissés ou délinquants, d'après le principe: éducation et travail. » Et elle ajoute: " le tribunal juge selon sa conscience, comme un bon père de famille, dans l'amour pour la vérité et la justice, et toujours dans l'intérêt des mineurs ». Cette définition va être en partie modifiée, notamment en fonction de la reclassification des mineurs opérée en 1925, comme en témoigne la définition du statut judiciaire de 1928 qui assigne par ailleurs un nouvel objectif aux tribunaux pour enfants - leur correction:

Dans le but de préserver, protéger, défendre et corriger les mineurs en danger 《moral, indisciplinés et délinquants, il y aura dans chaque division judiciaire une tutoria da infância, tribunal essentiellement d'équité, qui juge selon sa conscience dans l'intérêt des mineurs. "

C'est le caractère " équitable " de cette juridiction qui aurait permis à certains magistrats de contourner légalement le critère strict du droit pénal commun pour mieux tenir compte du comportement de l'enfant. C'est en tout cas ce que revendique Pedro Castro, président du tout jeune tribunal central pour enfants de Lisbonne, dans sa communication au congrès international de protection de l'enfance de 1913, à Bruxelles, où il fut officiellement invité à présenter l'expérience portugaise:

Le texte sec et rigide de la loi, qui applique indifféremment et impérative《ment une peine déterminée à chaque catégorie de faits criminels, disparaît une fois pour toutes pour faire place à la conscience du juge libre et paternel ${ }^{22}$. "

L'expression tutoria da infância renvoie à l'une des caractéristiques du modèle portugais. Afin de mieux faire ressortir la dimension tutélaire de la nouvelle juridiction, la LPE renonce au terme « tribunal pour enfants » pour adopter celui de "tutelle de l'enfance ", traduction littérale de tutoria da infância. Car il fallait tout faire, selon l'exposé des motifs, pour que «l'enfant ne soit pas marqué à vie par le stigmate d'avoir purgé une peine ", en se donnant comme but, "plutôt que punir au sens banal du terme celui de prévenir et guérir ». C'est la raison pour laquelle la justice était appelée à élargir son champ d'intervention, en s'intéressant non seulement aux mineurs délinquants, mais aussi aux mineurs délaissés ou en danger moral. Dans ce sens, l'usage du mot 
«tribunal " apparaissait aux yeux du législateur comme un contresens. Ce n'est qu'en 1944 que le terme " tribunal pour enfants " remplace définitivement celui de «tutelle de l'enfance». Mais pour une question de facilité, c'est le terme courant de tribunal pour enfants que nous emploierons au long de ce travail. L'adoption du mot " tutelle " pour désigner ce qui était bien un tribunal contribue à brouiller l'image de la nouvelle juridiction, que beaucoup prennent à ses débuts pour une institution de bienfaisance.

La LPE met en place deux types de tribunaux: les tribunaux centraux, dans les divisions judiciaires (comarcas) correspondant aux trois principales villes du Portugal - Lisbonne, Porto et Coimbra - et les comarcãos correspondant à chacune des divisions judiciaires restantes. Leurs attributions, qui initialement sont communes aux deux types de tribunaux, vont se différencier à partir de 1925. Ces derniers sont alors considérés comme auxiliaires des tribunaux centraux. C'est ainsi que nous les désignerons à partir de maintenant. Il s'agit dans les deux cas de tribunaux collectifs fonctionnant donc sur un mode collégial. Ils sont constitués par un président, choisi parmi les magistrats de carrière (il n'y a pas de magistrats spécialisés au Portugal), sauf dans le cas du tribunal central de Coimbra, où, jusqu'en 1935, cette fonction est tenue, pour des raisons que nous ignorons, par le professeur de droit pénal de l'université de la même ville, et deux juges assesseurs: un médecin et un enseignant du secondaire qui en province pourra être remplacé par un instituteur. Les juges assesseurs ont fondamentalement un rôle d'expertise et ils interviennent dès que l'enfant est déféré devant le tribunal, pendant la phase préparatoire du procès. Le mineur comparaît rarement devant le tribunal, il est entendu séparément par le président, ce qui est une façon de neutraliser l'effet intimidant de la cour collégiale $^{23}$. Ce type de fonctionnement vise à sauvegarder la dimension pluridisciplinaire de la justice des mineurs, que le père Antonio de Oliveira n'a de cesse de défendre dans ses ouvrages. Commentant, par exemple, le discours de Carton de Wiart au premier congrès international de protection de l'enfance, où la collaboration entre le juridique et le médical fut largement défendue, il prône, à l'instar du ministre belge, la nécessité de joindre à ce diptyque, la "science de la pédagogie $e^{24}$ ». Ainsi en 1919, dans l'exposé des motifs de ce qui reste le dernier texte de loi élaboré sous son autorité, même s'il a partiellement avorté (le décret 6117 du 20 septembre), lit-on:
23. Oliveira Augusto, Protecção moral e juridica, Lisbonne, 1929, p. 39-40.

24. Oliveira Padre Antonio de, Deixemos os..., op. cit., p. 415. 
25. La généralisation du juge unique à l'ensemble des tribunaux n'interviendra qu'en 1962.
Les bases de la jurisprudence relativement aux enfants ne peuvent se trouver

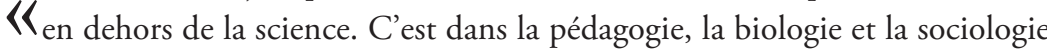
que se fonde réellement le nouveau droit. "

Puisant dans ce savoir multiforme sur l'enfant, la justice des mineurs est d'après lui plus à même d'accomplir la tâche que lui assigne la LPE:

Le premier gouvernement de la République, pour libérer l'enfant du vieux $\ll_{\text {empirisme du droit pénal, l'a confié aux lois naturelles de la science de }}$ l'éducation. La lutte suprême du progrès se déroule dans le champ éducatif. L'éducation est le grand champ de bataille (en français dans le texte original). Ainsi affirme Littré. »

Or, si personne ne remettait en cause l'importance d'une approche pluridisciplinaire, notamment dans la phase d'instruction, la collégialité des tribunaux est devenue rapidement un objet de controverse, d'autant qu'elle se situait à contre-courant de la tendance dominante dans les pays étrangers. Ainsi, à partir de 1928, seuls les tribunaux auxiliaires vont continuer à avoir une organisation collective, malgré l'intérêt de plus en plus marqué par le juge unique ${ }^{25}$. Pour des raisons budgétaires, sa généralisation n'est pas envisageable car cela obligerait à ce que chaque tribunal auxiliaire dispose d'un refuge pour l'observation des mineurs. L'organisation collective vient suppléer d'une certaine manière, comme le remarque le décret 10767 dans l'exposé des motifs, les ressources offertes par « les laboratoires d'examen, d'observation et d'enquête que sont les refuges » et dont les tribunaux centraux sont les seuls à disposer. Pour les mêmes raisons, les tribunaux auxiliaires ne bénéficient pas de la plénitude de juridiction leur permettant de prescrire toutes les mesures en faveur des mineurs, contrairement aux centraux. Ils ne sont autorisés à prescrire que celles n'exigeant pas une procédure préalable d'observation et d'examen et qui au fond se réduisaient aux mesures d'internement dans les établissements de réforme ou de correction pour mineurs délinquants ou assimilés. Dans ces circonstances, lorsqu’un tribunal auxiliaire juge nécessaire de procéder à l'internement d'un mineur, il doit proposer son transfert vers un tribunal central pour être examiné et observé dans le refuge avant d'être jugé. Relativement aux autres mesures dites « à caractère local » applicables aux mineurs délinquants, comme le blâme ou la liberté surveillée, il n'y a pas de restriction juridictionnelle. De même, il n'y a pas de 
restriction d'aucune sorte en ce qui concerne les mineurs en danger moral et, dans certains cas, les indisciplinés.

Les tribunaux pour enfants ont pour objectif de juger les causes civiles et pénales des mineurs des deux sexes, de moins de 16 ans, que la LPE divise en quatre groupes du point de vue juridico-social comme base des décisions des tribunaux: mineurs en danger moral, délaissés, délinquants et indisciplinés. Cette classification, sur laquelle nous reviendrons, ainsi que les limites d'âge vont évoluer par la suite. Signalons dès maintenant les changements relatifs à l'âge. Contrairement au souhait de l'auteur de la LPE (voir l'exposé des motifs) qui préconisait 18 ans, et pour tous les mineurs, la compétence des tribunaux pour enfants ne sera élargie postérieurement qu'aux mineurs en danger moral et aux indisciplinés, et ceci jusqu'à 21 ans (majorité civile). Parallèlement, à partir de 1925 , les jeunes de 16 à 18 ans condamnés par les tribunaux ordinaires à certains types de peines pourront éventuellement être autorisés à purger leur temps de prison dans une colonie correctionnelle pour mineurs délinquants de moins de 16 ans. Enfin, le projet de création d'une prison-école, selon le modèle Borstal, pour jeunes délinquants de 16 à 21 ans, apparaît, en 1934, comme la dernière pierre de l'édifice qu'est la juridiction tutélaire. Il passe, cependant, dès 1936, et avant même d'être promulgué, sous la tutelle de l'administration pénitentiaire. L'âge limite jusqu'auquel les tribunaux pour enfants doivent être compétents constitue un thème très débattu par les spécialistes, comme par exemple au cours du congrès de l'AIPE, réuni à Lisbonne, en 1931.

La division des mineurs en quatre groupes est l'objet de nombreuses critiques, en raison de son manque de rigueur juridique ou de ses redondances, au point que les juges renoncent, semble-t-il, parfois, à indiquer le régime d'internement dans leurs décisions à cause de la difficulté à s'y retrouver. Passons rapidement en revue les critères employés par la LPE pour définir chacun des groupes. La classification des mineurs en danger moral repose sur une longue liste de situations sociales vues comme des menaces réelles ou potentielles pour leur intégrité physique ou morale, du fait des conditions de vie ou du comportement des parents ou tuteurs: incarcération, incapacité, abandon, mauvais traitements, misère, mendicité, vagabondage, immoralité, métiers dangereux ou inhumains, etc. De cette liste, "limitative et non illustrative " comme le remarque Augusto de Oliveira, la LPE dégage trois sous-groupes: abandonnés, pauvres et maltraités. Or, faire des " pauvres " une sous-catégorie à part, c'est 
comme si a priori les abandonnés ou maltraités ne l'étaient pas. Le groupe des mineurs délaissés, dont le terme prête aussi à confusion, ce qui contribue à sa disparition en 1925, comprend ceux qui vivent habituellement dans l'oisiveté, le vagabondage, la mendicité ou le libertinage, de façon isolée ou en compagnie d'autres individus. La loi donne de ces pratiques des définitions souvent inattendues, comme celle qui identifie le mineur oisif à celui qui vit chez ses parents, tout en se montrant " réfractaire à l'idée d'instruction ou de travail sérieux et utile ". Ou encore, celle qui considère comme vagabond (vadio) le fugueur qui vit de la mendicité ou du vol, etc. Quant au libertinage, le terme recouvre principalement les activités liées à la prostitution, à sa pratique ou son exploitation, etc., mais il concerne aussi la fréquentation de salles du jeu ou le goût immodéré pour "l'animatographe ", l'une des obsessions de l'époque chez les criminologues. La classification des mineurs délaissés repose ainsi sur une série de modes de vie irréguliers liés à l'entourage, mais également, ce qui est nouveau, nous semble-t-il, à l'individualité du mineur (pour ne pas employer celui de personnalité encore peu utilisé à l'époque), comme lorsque la loi évoque ses " tendances immorales ou criminelles manifestes » ou sa " répugnance instinctive pour l'instruction et le travail». On pourrait attribuer cette lecture à la tendance vers la psychologisation de la délinquance qui commence à s'affirmer. Mais cela serait réducteur. Car ce qui distingue, à la lumière des critères indiqués par la loi, un mineur "délaissé » d'un mineur "abandonné " (deux adjectifs qui du point de vue lexical sont synonymes), c'est le défi aux normes sociales ainsi que la façon de déjouer les attentes sociales que nous révèle le comportement du premier mais pas nécessairement le second. On comprend mieux dans ce cas pourquoi, suite à la disparition de la catégorie des mineurs délaissés, comme nous le verrons, c'est autour de ceux censés présenter des «tendances criminelles ou immorales " qu'un nouveau concept de délinquance associé aux modes de vie récalcitrants et non à un fait criminel précis va prendre corps. Quant au groupe des mineurs indisciplinés, il comprend les enfants de la correction paternelle, introduite dans le code civil de 1867, et les mineurs " incorrigibles » des établissements d'assistance et de bienfaisance. Enfin, le groupe des mineurs délinquants regroupe les auteurs de crimes et de contraventions au sens du Code pénal (à remarquer qu'au Portugal, crime et délit sont synonymes sur le plan juridique) mais que la LPE considère, eux aussi, comme victimes, lorsqu'elle écrit, dans l'exposé des motifs: "Produits 
inconscients du milieu, de l'hérédité [...], ils doivent être jugés selon l'esprit pondéré du magistrat plutôt que selon la lettre inflexible des codes. "

Arrêtons-nous justement sur la place des parents dans le cadre de cette réforme, en nous limitant aux dispositions de la LPE, dans la mesure où celles-ci sont à la fois complétées et simplifiées par la suite, à plusieurs reprises. Signalons uniquement l'adoption, en 1925, de la pension alimentaire, en tant que devoir complémentaire des parents ou des tuteurs à l'égard des enfants mineurs. La phrase citée reprend à sa manière l'idée qui sous-tend historiquement la justice des mineurs, selon laquelle l'enfant est une victime à protéger plutôt qu'un coupable à punir ${ }^{26}$. D'après Renouard, ce qui marque ce changement de représentation est le fait que la famille, et les parents en particulier, deviennent responsables potentiels du comportement irrégulier des enfants. Ainsi, on déculpabilise partiellement le mineur pour culpabiliser la famille ${ }^{27}$. C'est ce que confirme le processus de classification des mineurs en danger moral, basé en particulier sur les risques liés au comportement des parents. Mais la LPE va plus loin, en établissant plusieurs formes d'inhibition de la puissance parentale ou tutélaire, qui représentent pour le père Antonio de Oliveira " la première brèche dans l'édifice de l'ancien patria potestas » consacré dans le Code civil ${ }^{28}$. La déchéance parentale intervient dans les cas où la négligence avérée, les mauvais exemples, les formes de cruauté, la spéculation ou la délinquance du père, de la mère ou du tuteur compromettent la santé, la sécurité ou la moralité des enfants. Mais elle intervient également suite à l'internement du mineur dans une maison de correction ou à son placement dans une famille d'accueil, afin de garantir l'application de ces mesures, qui révéleraient en creux la faillite des parents. Le durcissement des conditions permettant que les enfants puissent être internés dans une maison de correction à la demande du père va dans le même sens. Alors que le Code civil autorise ce dernier à faire interner un fils "désobéissant ou incorrigible " pendant une durée maximale de trente jours et à y mettre un terme à tout instant, avec la LPE, la durée de l'internement, qui entretemps avait déjà été allongée, passe à six mois, sans possibilité de reprendre l'enfant avant le terme prévu. Car il convient de mettre fin à ce qui apparaissait comme un abus de la part de certains parents, accusés par exemple de faire bénéficier leurs enfants d'une formation professionnelle aux frais de l'État, tout en se permettant de les récupérer quand bon leur semble. À l'empathie, teintée de paternalisme, à l'égard des mineurs, qui traverse la LPE, s'oppose le ton
26. SudAn Dimitri, « Da criança culpada ao sujeito de direitos: alterações do modo de gestão da delinquência infantil (1982-1989) ", Infância e Juventude, 3, 1997 p. 73

27. Cité dans « Da criança culpada... », p. 79.

28. Olivelra Padre Antonio de, Intimidação, Lisbonne, s. d., p. 237. 
29. SAnto João Miguel R. S., "Crianças malfeitoras" a contas com a justiça, Lisbonne, Universidade de Lisboa, 2000. accusatoire, aux accents moralisateurs, vis-à-vis des familles, comme nous le montre cet extrait de l'exposé des motifs:

Abandonnés à eux-mêmes (les enfants), sans parents, ou avec une famille 《et des parents qui les poussent vers le vice ou la perversion; remis dans les mains de parents ou de tuteurs qui en raison de leur pauvreté ne peuvent pas les éduquer ou qui les transforment en martyrs innocents de la férocité de leurs instincts irrépressibles; en les maltraitant, en pratiquant des injustes et dangereuses différences de traitement au sein de la fratrie, en les obligeant à se livrer à la mendicité et au vagabondage: si cela ne peut pas être considéré comme criminel, c'est bien à devenir criminels qu'on est en train de les préparer. D'où, l'obligation de l'État ou de toute personnalité dûment autorisée de les arracher à ce milieu corrupteur, en les tenant sous leur tutelle jusqu'à leur émancipation...»

Voilà pourquoi il semble inadéquat, précise la loi, d'appeler " tribunal » une juridiction qui se destine à des enfants qui sont avant tout victimes de leur milieu. Malgré un échantillonnage extrêmement réduit - 35 procès de mineurs jugés dans le tribunal central pour enfants de Lisbonne entre 1920 et 1930 -, la recherche de João Miguel Santo ${ }^{29}$ apporte un autre éclairage sur le contexte familial dans lequel s'inscrivent les pratiques délictueuses de ces mineurs. Si le vol, le vagabondage et la prostitution (féminine) sont les délits les plus représentés, la lecture des procès permet de constater l'association presque systématique du vol au vagabondage. Celui-ci apparaît comme:

le comportement le plus courant chez les enfants issus des familles les plus 《 défavorisées dont les parents n’avaient pas la possibilité de veiller et de contrôler l'éducation et particulièrement la scolarité, en raison de leur activité professionnelle. La rue apparaît ainsi comme le milieu de perversion par excellence. [...] Ces mineurs arrivaient au tribunal principalement par le biais des membres de la famille les plus proches. N'ayant pas pour la plupart la possibilité de les contrôler, ils se tournaient vers l'institution judiciaire pour qu' elle puisse les relayer sur le plan éducatif».

L'auteur conclut que le profil de ces mineurs, dont il faut souligner que la large majorité avait une activité professionnelle, surtout en tant qu’apprentis, ne 
serait finalement pas très différent de « ce que les portraits des familles ouvrières de l'époque nous révèlent indirectement ${ }^{30}$ ". Ainsi, " la tendance des parents à transférer la charge et la subsistance des mineurs vers l'État ${ }^{31}$ ", plutôt qu'une forme d'abandon ou de démission, serait un signe d'intérêt pour leur avenir, face aux difficultés quotidiennes qui les accablent.

Les discours sur l'hérédité comme facteur de dégénérescence accentuent cette tendance à la culpabilisation des parents. Il s'agit d'un thème récurrent chez le père Antonio de Oliveira qui voit là une menace à la survie des générations, à ce qu'il appelle la « race ». Cette appréhension n'est pas que fantasmatique. Les ravages liés à la tuberculose, à la syphilis ou à l'alcoolisme étaient alors bien réels. Le ton hygiéniste de son discours était une façon de décliner la nécessité de protection à l'égard des enfants. C'est sous cet angle-là, que dans l'exposé des motifs, la LPE attribue à son tour comme mission à la justice des mineurs, la prescription d'un "processus de thérapeutique morale, d'hygiène préventive du crime et avant le crime et d'hygiène curative contre le crime consommé pour éviter sa répétition ". On retrouve la même analyse, dans des termes presque identiques, dans le premier ouvrage du père Antonio de Oliveira, à propos des conséquences sur la médecine des découvertes de Pasteur qui ont permis d'établir " un nouveau plan de défense contre la maladie ». D'un côté, pour empêcher son éclosion ("hygiène préventive») et, de l'autre, pour secourir le malade tout en évitant sa propagation (" thérapeutique préventive »). Il s'agirait d'un plan identique à celui de la criminologie positiviste contre le crime (il parle d'ailleurs de " corrélation étroite " entre Pasteur et Lombroso), avec une étiologie de la criminalité et une classification des criminels servant de base à la diversité des « remèdes " à leur administrer ${ }^{32}$.

Il va jusqu'à considérer le modèle hygiéniste contre la maladie comme plus pertinent que celui d'Adolphe Prins contre le crime en matière de défense sociale, dans la mesure où celui-ci ignorerait la question de l'hérédité. Prenant comme exemple la figure d'Oswald, ce personnage d'Ibsen qui ne cessera pas de le hanter dès qu'il a assisté à la représentation de la pièce du dramaturge norvégien dans un théâtre de Lisbonne ${ }^{33}$, il écrit à ce propos: «La solution de Prins n'allant pas jusqu'aux origines premières du mal laisse en vie, en permanente activité, tous ces horribles spectres qui engendrent des dégénérés comme Oswald, qui passent leur vie [...] à payer les péchés des parents ${ }^{34}$."

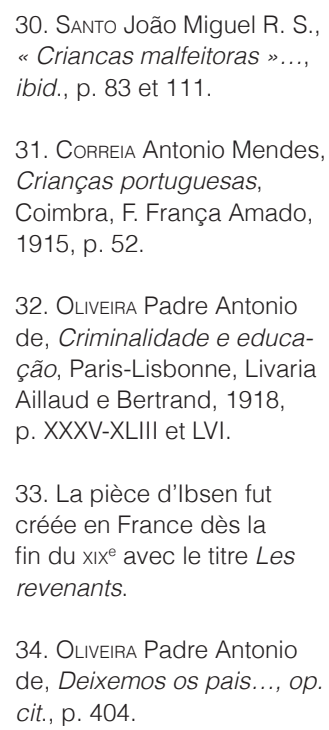


35. Santos José Beleza dos, « Regime juridico dos menores delinquentes em Portugal », Boletim da Faculdade de Direito da Universidade de Coimbra, $n^{\circ} 71-80,1923-1925$, p. 156-157.

36. Gersao Eliana, "Menores agentes... ", op. cit., p. 404.
Ayant pour toile de fond l'image de l'enfant victime, la LPE ouvre le chemin à la dépénalisation de la délinquance des mineurs de moins de 16 ans, dont la première étape est la remise en cause de l'idée de discernement comme critère de responsabilité pénale. Jusque-là, le Code pénal admettait l'irresponsabilité absolue des mineurs de moins de 10 ans ainsi que celle des plus de 10 ans et moins de 14, s'ils avaient agi sans discernement. Quant aux mineurs de 10 à 14 ans ayant agi avec discernement, ils étaient soumis aux peines de droit commun, même s'ils avaient la possibilité de bénéficier d'une réduction en termes de durée. Au-delà de 14 ans, il n'y avait pas lieu de s'interroger sur l'existence ou non de discernement: les mineurs ne pouvaient compter que sur une atténuation de la rigueur pénale, traduite dans la dispense ou l'allègement de certaines peines $^{35}$. La LPE finit avec toutes ces distinctions, en amorçant une rupture avec la logique pénale, sans s'en détacher totalement. D'abord, en baissant d'une année (9 ans à la place de 10) la limite de l'irresponsabilité absolue au-dessous de laquelle aucune peine ne pourrait être prononcée. Quant aux mineurs de 9 à 16 ans, la LPE prévoyait une gradation des mesures en fonction de la tranche d'âge (9-13 ou 13-16 ans) et des peines de prison correspondant aux délits commis. Parmi les mesures prévues dans les cas les plus légers, en plus de l'internement dans un établissement de réforme, d'éducation ou d'assistance, l'une (le placement en famille d'accueil) était inédite au Portugal et l'autre (la liberté surveillée) relativement nouvelle car peu appliquée jusque-là. Dans les cas les plus graves, les mesures pouvaient aller jusqu'à la détention mais à purger dans un établissement de correction exclusivement. C'était la fin des peines de prison pour les mineurs de moins de 16 ans que l'on peut considérer comme l'amorce de la dépénalisation de la criminalité infantile que le décret 10767 du 15 mai 1925 rendra effective. À partir de cette date, aucune des peines établies dans le Code pénal, et non seulement l'incarcération, ne pourra plus être appliquée à un mineur de moins de 16 ans. Gersão voit dans cette " conquête ", l'acquis le plus significatif du modèle portugais en matière de justice des mineurs - une « donnée culturelle» qu'il faudra à tout prix préserver ${ }^{36}$.

Entre hésitations et attentisme, doutes et discussions, la mise en place de la justice des mineurs va connaître un ralentissement considérable après l'enthousiasme des débuts et la mise en place assez rapide du tribunal central de Lisbonne, précédée de celle du refuge annexe. Faute d'un édifice convenable, ce dernier, qui va fonctionner dans les installations de l'ancien dépôt dont 
il est l'émanation, démarre sans la section féminine. De la même manière, les garçons classifiés comme délaissés et délinquants, de 14 à 16 ans, ont dû être internés provisoirement dans l'espace anciennement réservé aux mineurs de la prison centrale de Lisbonne. Si l'installation du tribunal central pour enfants de Porto a lieu dès 1912, celui de Coimbra, qui démarre en 1927, ne commence à fonctionner pleinement qu'en 1935. Quant aux tribunaux auxiliaires, leur installation va avoir lieu en 1925 couvrant la totalité des divisions judiciaires dès l'année suivante. Relativement aux refuges, limités finalement aux trois tribunaux centraux, il faudra attendre les années 1927-1928 pour que l'ensemble de leurs sections, masculines et féminines, soient en état de marche, celles de Lisbonne et de Porto pour les garçons étant les seules à avoir démarré partiellement presque en même temps que les tribunaux. Mais voir dans cette lenteur le résultat d'une simple inertie administrative serait erroné. D'autres facteurs, bien plus importants, sont à relever, comme la crise sociale, politique et économique sans précédent dans laquelle la première grande guerre plonge le Portugal, obligeant à de sévères restrictions budgétaires. De ce fait, l'instabilité chronique qui marque dès ses débuts le régime républicain sur le plan social et politique ne pouvait qu'empirer ${ }^{37}$. Enfin, les débuts laborieux des deux premiers tribunaux, qui se trouvent vite saturés, dûs à un manque cruel de relais (nombre de places insuffisant dans les établissements de justice et d'assistance, service social embryonnaire, initiative privée quasi inexistante, absence de personnel qualifié, etc.), contrariaient forcément les meilleures volontés. Il faut attendre 1919 pour que la justice des mineurs gagne un nouveau mais relatif élan, avec une série de mesures importantes promulguées au cours de l'année (les dernières inspirées par le père Antonio de Oliveira), mais qui resteront en grande partie sans suite. L'ouverture de deux nouvelles écoles de réforme est l'un des rares acquis de l'ambitieux programme sur les établissements de réforme et de correction alors promulgué, d'autant qu'il s'agissait d'un volet quelque peu délaissé par la LPE. Sur un autre plan, une mesure marquante est la création de l'inspection générale des services de protection des mineurs, première instance de régulation et de coordination indépendante des services pénitentiaires, dont la direction fut confiée au père Antonio de Oliveira. De santé fragile, celui-ci meurt en 1923, trois ans à peine après son démarrage. Dans la préface à l'un de ses ouvrages, écrite quelques mois avant sa mort, il ne cache pas son amertume face aux malentendus que la réforme de 1911 continuait à susciter, notam-
37. Pour une vue d'ensemble sur la Première République, voir l'excellent recueil coordonné par Rosas Fernando et Rollo Maria Fernanda, Historia da Primeira Republica portuguesa, Lisbonne, Tinta da China, 2010. 
38. Deixemos os... p. LXVII. Contrairement aux magistrats, le père Oliveira a bénéficié d'un soutien appuyé d'un certain nombre de noms illustres de la médecine et de la psychiatrie portugaise.

39. Voir le Relatorio do juiz presidente da tutoria central da infância de Lisboa. ano judicial de 19141915, Lisbonne, Imprensa Nacional, 1916, p. 29 et l'exposé des motifs du décret du 15 mai 1925. ment parmi les magistrats, tout en avouant sa joie de voir les idées qui l'avaient inspirée consacrées dans les congrès internationaux et intéresser un nombre croissant de pays étrangers ${ }^{38}$. Deux d'entre eux doivent être mentionnés en raison des répercussions effectives de la LPE dans leurs législations: l'Italie qui adopte assez rapidement une grande partie de ses dispositions et le Brésil qui s'en inspire largement pour l'organisation, entre 1923 et 1924, des juizos de menores (" tribunaux pour mineurs ") ${ }^{39}$. C'est l'avocat Augusto de Oliveira qui remplace le père Antonio de Oliveira à la tête de l'inspection générale. Celle-ci va devenir dix ans plus tard la direction générale des services juridictionnels des mineurs dont il assume également la responsabilité jusqu'en 1939. Son action se distingue par une intense activité internationale en tant que représentant du Portugal au sein de l'AIPE et du Comité consultatif pour la protection de l'enfance et de la jeunesse de la Société des Nations. Élu vice-président de l'AIPE, au congrès de Rome de 1926, il succède à Henri Rollet, dont il est proche, à la présidence de cette organisation, lors du congrès de Lisbonne de 1931. Augusto de Oliveira a exercé également les fonctions de secrétaire général de l'Association internationale des juges pour enfants. Si ce parcours est un signe évident de son prestige personnel, il n'en témoigne pas moins du rayonnement du Portugal dans les milieux internationaux de protection de l'enfance à cette époque.

Il appartient donc à Augusto de Oliveira de mener à son terme le processus d'institutionnalisation de la justice des mineurs, dont le décret 10767 du 15 mai 1925, qui porte sa marque, est une étape décisive. Comme son intitulé l'indique - règlement général des services juridictionnels et tutélaires des mineurs (SJTM) -, celui-ci procède à une profonde réorganisation de ces services qui présentent, selon les termes même de l'exposé des motifs, un certain nombre de dysfonctionnements auxquels il est urgent de remédier: fonctions mal définies, absence d'articulation avec d'autres services publics, comme l'éducation et l'assistance, etc. C'est en février 1924 que le ministre de la Justice soumet à l'approbation de la Chambre des députés la réorganisation de ces services. Mais il est probable qu'elle ait été accélérée du fait de la déclaration de Genève du 26 septembre de la même année, dont le Portugal est l'un des pays signataires, qui sera adoptée en tant que charte des droits de l'enfant par la Société des Nations. Rappelons que l'un des objectifs de la réglementation des SJTT est l'établissement, dans un texte plus systématisé, du schéma d'un futur Code de l'enfance qui réunirait l'ensemble de la législation sur les mineurs, permettant 
une meilleure prise en compte des besoins de l'enfant et une meilleure synergie entre les services concernés. Ce qui ne correspond pas tout à fait à l'objectif assigné par le décret de 1919 qui prévoit déjà l'organisation d'un Code de l'enfance dans le but de "juger les mineurs en danger moral, délaissés et délinquants, conformément à l'esprit et aux principes de la LPE ». Il s'agit d'un projet cher au père Antonio de Olivera qui trouve scandaleux que les mineurs, malgré l'existence d'une juridiction spéciale, continuent d'être assujettis au droit pénal commun. Au point, semble-t-il, de s'être lancé lui-même dans la rédaction de ce code sans avoir pu le terminer ${ }^{40}$. Dans le même ordre d'idées, le ministre de la Justice annonce, dans un entretien au quotidien O Século du 29 décembre 1919, qu'une commission chargée d'élaborer le Code de l'enfance va bientôt être nommée avec la finalité de "soustraire les mineurs à la pénalité des adultes ». Il revient cependant au décret 10767 de mener à bien cette tâche, le Code de l'enfance ne voyant jamais le jour.

Ce décret, dont l'importance dépasse la simple réglementation des SJTM, apporte quelques inflexions majeures au cadre inaugural dont il partage néanmoins les principes fondamentaux. Il cherche à tenir compte de l'expérience des premières années de fonctionnement de la justice des mineurs ainsi que des conclusions des congrès internationaux de protection de l'enfance et des tribunaux pour enfants réalisés depuis la promulgation de la LPE (voir l'exposé des motifs). Aussi, par ses incidences sur le concept de délinquance, élargi dans un sens plus subjectif, l'adoption de la classification, partiellement remaniée, des mineurs introduite en 1911 est particulièrement importante. Le groupe des mineurs délaissés (desamparados) disparaît, entraînant une reconfiguration des groupes des mineurs en danger moral, délinquants et indisciplinés qui, eux, sont maintenus. Rappelons que la LPE classifie de délaissés les mineurs qui se livrent de façon habituelle à l'oisiveté, au vagabondage, à la mendicité ou au libertinage, en raison d'un certain nombre de situations sociales et de facteurs individuels, dont l'existence de «tendances immorales ou criminelles manifestes ». Or, c'est justement l'existence ou non de ces tendances qui va déterminer le choix du groupe auquel les ex-mineurs délaissés seront désormais rattachés: les vagabonds et libertins (le texte de loi ne cite plus les mendiants ni les oisifs, comme s'ils se confondaient désormais avec les premiers) présentant des " tendances criminelles définies et confirmées ", de même que les indisciplinés, sont assimilés aux délinquants au titre des mesures devant leur être
40. Magalmaes José, «Padre Antonio de Oliveira ", Boletim do Instituto de Criminologia, vol. III, $2^{\mathrm{e}}$ semestre, 1922. 
41. Oliveira Augusto «Relatorio sobre o funcionamento dos tribunais de menores em Portugal », Protecção..., op. cit. p. 35-52

42. L'auteur désigne pour " état de délinquance » ou " prédélinquance», « la tendance innée ou la prédisposition longuement acquise pour la pratique du crime par des habitudes invétérées que le mineur révèle à travers son mauvais instinct ». Et il ajoute: « II ne faut pas confondre cet état individuel avec la nuisible et perverse prédisposition du milieu social du mineur qui, elle, ne devra donner lieu qu'à une reconnaissance du danger moral. " appliquées et les autres reconnus comme mineurs en danger moral. Il ne faut pas confondre cette classification juridico-sociale, qui sert de base aux délibérations des magistrats, avec les procédures de classification destinées à ponctuer la prise en charge des mineurs au sein du réseau spécialisé.

Dans son rapport au congrès de l'AIPE de Rome ${ }^{41}$, AGO revient longuement sur ce processus de reclassification qui ne donne lieu à aucune explication dans l'exposé des motifs du décret 10767 qui se limite à le reprendre. Dans une critique voilée à l'école pénale classique, l'auteur, que nous suivrons de près, voit dans l'introduction par la LPE du groupe des mineurs délaissés, dont il critique d'ailleurs le caractère redondant au regard des autres groupes, une concession à la "vieille " conception de criminalité: "On a vu le délit, là où il fallait voir le délinquant. On a continué à voir le crime comme entité abstraite, alors qu'il aurait fallu évaluer et examiner un état de délinquance, surtout s'agissant de jeunes ${ }^{42}$. " Ce sera cet argument à la bonne manière positiviste qui va servir de fil conducteur à sa démonstration faite à partir de deux exemples. Il évoque d'abord le cas des nombreux mineurs qui s'adonnent à la mendicité, au vagabondage ou au libertinage sans que ces pratiques deviennent généralement un « mode de vie » auquel ils seraient liés par « plaisir » ou par « volonté délibérée ». Il s'agit de mineurs dont l'expérience tendrait par ailleurs à prouver qu'ils n'ont pas de tendances criminelles. Dans ces circonstances, les mesures à prescrire ne peuvent être que celles prévues pour les mineurs en danger moral car c'est bien ce qu'ils sont en réalité. Ce n'est que lorsqu'on a affaire à des habitudes invétérées et que cela coexiste avec des tendances accentuées pour le crime, que les mineurs doivent être considérés sinon comme délinquants, au moins comme prédélinquants, afin d'être soumis aux « mesures thérapeutiques de rééducation, réforme ou correction » ainsi qu’au régime juridique propre aux délinquants. Et ceci, même si aucun acte criminel précis ne peut être prouvé devant le tribunal. AGO cite ensuite le cas d'un mineur ayant commis un crime grave au regard du Code pénal, alors qu’il ne présente pas de " tendances définies et accentuées » ou de " prédispositions innées ou longuement acquises pour la pratique du crime ». Dans ce contexte, le seul fait d'avoir pratiqué un acte criminel, malgré sa gravité, n'apparaissait pas comme une raison suffisante pour classifier son auteur comme délinquant, son geste devant être considéré comme accidentel. De même, il arrive que le fait criminel pour lequel un mineur est poursuivi devant la justice ne puisse pas être prouvé en tribunal, alors que l'examen et l'observation dont 
il a fait l'objet montrent qu'il souffre « d'un état de délinquance défini et accentué ", devant pour cette raison être considéré comme délinquant. Nous voilà donc face à une approche de la délinquance à l'opposé de celle généralement admise et qui privilégie la manière d'être au détriment de l'acte ou la personnalité de l'infracteur au détriment de l'infraction. Ce qui constitue une remise en cause radicale de l'un des principes de base du droit pénal commun, selon lequel il n'y a pas de crime ou délit sans violation d'une disposition pénale. Or, pour Augusto de Oliveira, continuer à adopter une vision si stricte de la délinquance ne peut que porter atteinte à l'identité même de justice des mineurs, à savoir son caractère tutélaire, en s'interdisant par exemple d'intervenir auprès d'un enfant qui n'a pas commis de délit mais chez lequel on décèle l'existence d'un "état de délinquance ». Non par une exigence de prévention à proprement parler, car celle-ci est censée viser les facteurs du milieu susceptibles de pervertir le mineur, mais de traitement, puisque le " mal » est déjà là, d'où l'identification de la prédélinquance avec la délinquance même. Au-delà de la dépénalisation, qui vise à contourner les principes du droit pénal commun sans les remettre en cause, il s'agit désormais de considérer la délinquance infantile non pas « objectivement ", selon ces principes, mais " selon un critère essentiellement subjectif, d'accord avec les principes de la criminologie moderne ", comme le précise Augusto de Oliveira à une autre occasion ${ }^{43}$. Il fait ici référence au déplacement opéré par l'école pénale positiviste et le mouvement d'individualisation de la peine à la fin du XIX ${ }^{\mathrm{e}}$ siècle tendant à privilégier la personnalité du délinquant contre l'infraction criminelle. Délesté de ses outrances positivistes d'origine, ce déplacement est au cœur de la pensée de défense sociale. L'auteur insiste à plusieurs reprises sur l'importance des procédures rigoureuses d'observation et d'examen dans l'identification d'un état de délinquance ou de prédélinquance. Un concept qui, en raison de l'indétermination de ses contours, présente tout de même le risque potentiel d'éveiller indéfiniment la logique du soupçon et du contrôle. Mais au-delà de cet aspect, les procédures d'évaluation ont d'après Augusto de Oliveira un rôle plus large à jouer, tant en ce qui concerne le choix du régime et des mesures à appliquer aux mineurs délinquants que dans leur propre processus de classification qui ne doit plus être laissé au seul arbitrage des tribunaux. Néanmoins, conscient des carences en matière d'observation et d'examen, tant au niveau des équipements que du personnel, il finit par avoir une position moins tranchée relativement au droit pénal commun. C'est ce
43. Oliveira Augusto, "Protecção... ", op. cit., p. 18. 
44. Voir à ce propos, DUPONTBOuchat Marie-Sylvie, " De la prison à l'école de bienfaisance. Origines et transformations des institutions pénitentiaires pour enfants en Belgique au xIX siècle (1840-1914) ", Criminologie, XXVIII, 1, 1995, p. 43-47. qui transparaît de l'arrêté du 18 mai 1927, signé de sa propre main, qui fixe les principes de classification des trois groupes de mineurs auxquels s'adresse la juridiction tutélaire. Ainsi, il est précisé que pour la définition d'un mineur de 9 à 16 ans comme délinquant:

la preuve des éléments objectifs du crime est moins pertinente que l'investi$\ll_{\text {gation des conditions d'ordre subjectif tendant à former l'intime conviction }}$ du tribunal de ce qu'il s'agit d'un criminel ou d'un délinquant, sans la stricte soumission aux règles (stricti juris) d'incrimination imposées par le droit pénal commun ».

Mais, revenant à un langage juridique plus " classique ", il ajoute que l'internement des mineurs délinquants dans les établissements de justice ne doit être proposé que face à des « délinquants d'habitude ou récidivistes et dans les cas les plus graves de délinquance primaire ». L'argument par lequel l'arrêté motive ce principe de classification traduit toute l'ambiguïté d'une démarche qui cherche par le même geste à punir et à guérir :

Il s'agit certes d'une mesure de défense sociale répressive du crime mais par 《le biais d'une juridiction tutélaire qui soumet simplement le délinquant à une action thérapeutique correspondant à l'étiologie criminelle mise en lumière. Elle passe par la rééducation morale, la formation professionnelle et le traitement des conditions psycho-pathologiques, endogènes et exogènes, observées ou confirmées lors de l'examen médical et combien de fois constatées à l'occasion de l'enquête auprès de la famille. »

Quant aux vagabonds et libertins avec des tendances criminelles assimilés aux délinquants et donc soumis aux mêmes mesures d'internement qu'eux, l'arrêté précise qu'en raison de leur degré de perversion ils peuvent présenter un caractère plus grave que les auteurs d'un crime pratiqué occasionnellement et sans antécédents connus. Il serait intéressant de savoir comment les tribunaux pour enfants se sont saisis de la nouvelle orientation issue de la disparition de la catégorie des mineurs délaissés. Dans la ligne de pensée de défense sociale, avec son effet de "triage " entre enfants en danger et pervertis ${ }^{44}$, elle aurait dû théoriquement permettre à de nombreux petits vagabonds d'échapper à la logique répressive, en les reconnaissant comme enfants en danger moral, tout 
en renforçant la dimension punitive à l'égard de ceux qui, du fait de leur assimilation aux délinquants, se voyaient soumis au même titre qu' eux à la mesure la plus sévère qu'est celle de l'internement dans un établissement de justice.

L'endurcissement à l'égard des petits vagabonds récalcitrants est indissociable du point de vue juridique du vide créé par la promulgation de la très répressive loi du 20 juillet 1912 sur le vagabondage (vadiagem) qui laisse en dehors de son champ d'application les mineurs de moins de $16 \mathrm{ans}^{45}$. Cette loi procède, en effet, à l'abrogation des articles du Code pénal qui déterminent le cadre juridique en vigueur dans ce domaine y compris pour les mineurs. En outre, c'est à ses dispositions que nous renvoie le décret 10767, lorsqu'il stipule que tout mineur considéré comme "incorrigible " (c'est dans ce sens uniquement, qu'à notre connaissance, et à l'exception d'un texte de loi avorté de 1919, l'idée d'incorrigibilité apparaît dans le droit pénal des mineurs au Portugal ou dans la littérature juridique y afférente), au moment (21 ans maximum) où il doit quitter l'établissement où il a été interné, peut être mis à la disposition du gouvernement. Selon les termes de la loi de 1912, il doit dans ces circonstances être interné dans une colonie pénale agricole ou envoyé, dans le cadre de la transportation pénale, vers l'Afrique. Nous ne pourrons pas nous arrêter sur le contexte socialement très perturbé (l'année 1912 commence avec la première grève générale du mouvement ouvrier portugais pourtant encore peu organisé), dans lequel la promulgation de cette loi intervient, la répression du vagabondage se confondant parfois avec celle des mouvements sociaux ${ }^{46}$. Quant aux mineurs, la présence permanente des petits vagabonds dans les rues des villes, et de Lisbonne en particulier, finit par discréditer la justice des mineurs, accusée d'inefficacité par la presse, à intervalles réguliers. Aussi, on peut trouver dans l'édition du soir du quotidien $O$ Século du 15 septembre 1925, ce titre éloquent: "L'augmentation de la criminalité infantile. Le tribunal pour enfants ne correspond pas aux objectifs pour lesquels il a été créé. »

Quelque peu en contradiction avec la place que lui-même accorde à la question, Augusto de Oliveira défend vers la fin de son rapport à l'AIPE la nécessité de réduire "le champ encore trop vaste de la délinquance infantile ", en s'intéressant davantage aux mineurs en danger moral. En raison de la crise que connaitrait la famille, il lui semble indispensable d'accorder aux tribunaux pour enfants de plus larges attributions à l'égard de ces mineurs. Pour cela, il faut commencer par abandonner la liste limitative de situations pouvant donner lieu

\author{
45. Sur la place de cette \\ loi dans le long processus \\ de construction du statut \\ juridique du vagabond, \\ voir FATELA João, « Les \\ milles visages du vadio \\ portugais », Guestin André \\ et Kalifa Dominique (éd.), \\ Les Exclus en Europe 1830- \\ 1930, Paris, Editions de \\ l'Atelier, 1999, p. 49-58. \\ 46. Voir à ce propos, FATELA \\ João, O sangue e a rua, \\ Lisbonne, Dom Quixote, \\ 1989, p. 232 sq.
}


à l'inhibition de la puissance parentale qui constituait l'essentiel de leur activité dans ce domaine. Même si le décret 10767 reste fondamentalement axé sur la délinquance, il témoigne de la recherche d'un plus grand équilibre entre les deux catégories de mineurs. D’abord, en cherchant à délimiter plus rigoureusement les champs d'intervention vis-à-vis des uns et des autres. L'exposé des motifs dénonce à ce propos la confusion dans laquelle les SJTM sont tombés dans le passé, en appliquant aux mineurs en danger moral des mesures d'internement prévues pour les délinquants. Ainsi, il ne se contente pas de rappeler la décision prise en 1919 qui interdit la présence de ce type de mineurs dans les refuges, à moins de disposer d'espaces séparés, pour ne pas les mettre en contact avec les délinquants. Il étend cet interdit aux établissements de réforme et de correction où leur présence sera désormais également interdite. Tout en donnant la priorité à des mesures comme la liberté surveillée ou le placement en famille d'accueil, il insiste pour que l'internement dans un établissement de bienfaisance, d'éducation ou d'assistance, où ils ont d'ailleurs la priorité sur d'autres mineurs, se fasse rapidement. Enfin, la classification de danger moral tend à couvrir l'absentéisme scolaire régulier et les abus relatifs au travail des mineurs dans l'industrie. Une double préoccupation que l'on retrouve dans les consignes de l'arrêté de 1927 aux tribunaux auxiliaires à propos des mineurs indisciplinés au titre de la correction paternelle. En stipulant que doivent être classifiés comme tels les mineurs "qui se comportent comme des rebelles et réfractaires au travail et à la discipline dans la famille et dans l'école ». Assimilés aux délinquants, ils peuvent comme eux être internés dans un établissement de réforme ou de correction, mais dans une section à part.

L'approche de la délinquance infantile dans un sens plus subjectif autour de la " personnalité " de l'enfant ou de l'adolescent, et de son éventuel état de prédélinquance, aboutit, comme nous l'avons vu, au renforcement des procédures d'observation, d'examen et d'enquête qui caractérise le décret 10767. Celles-ci jouent un rôle déterminant, notamment dans la prescription et la reconfiguration des modalités d'internement des mineurs délinquants dont ce décret établit le cadre général, de façon à évaluer à la fois leur degré de perversion et leur capacité à se corriger. Ce n'est pourtant pas au nom de la nécessité d'identifier un éventuel état de délinquance que le décret justifie l'importance de ces procédures d'évaluation, mais à partir des rapports supposés entre anormalité et délinquance auxquels l'exposé des motifs consacre une longue et fasti- 
dieuse analyse, en s'appuyant sur les travaux du II ${ }^{\mathrm{e}}$ congrès international de protection de l'enfance qui s'est occupé de la question et dont il reprend les conclusions. Vu l'existence d'un « contingent important » de délinquants parmi les enfants anormaux, il conviendrait, lit-on, « de soumettre sans délai à une observation rigoureuse sur le plan psychologique et psychiatrique les mineurs délinquants ». Certes, la présence parmi les mineurs reçus lors de l'ouverture des tribunaux pour enfants de Lisbonne et de Porto d'un certain nombre d' " anormaux pathologiques ", selon la terminologie de l'époque, a mis les magistrats devant une situation très embarrassante en raison du manque de relais spécialisés ${ }^{47}$. Mais outre le fait que le décret ne s'y réfère pas, on n’y retrouve pas non plus de décisions en adéquation avec la nature et l'ampleur du phénomène telles qu'elles se dégagent de l'exposé des motifs. Ce qui nous amène à penser que le rapprochement entre l'anormalité et la délinquance a pour objectif principal de signifier l'importance de l'approche psychologisante de la délinquance des mineurs, dont le décret 10767 marque indéniablement le tournant, parallèlement à sa médicalisation, non plus sous l'angle hygiéniste, comme dans le cas de la LPE, mais sous celui de la psychiatrie.

L'observation des mineurs fait partie du dispositif mis en place par la LPE qui accorde cette compétence au refuge annexe au tribunal (dans ce cas, celui de Lisbonne), à côté de la détention temporaire des mineurs. Elles resteront les fonctions principales des refuges qui plus tard serviront également pour de courtes détentions punitives dans des espaces séparés de ceux consacrés à l'observation. D'après le premier rapport d'activité du tribunal central de Lisbonne, la procédure d'observation et d'examen est assez sommaire. Après son arrivée au refuge et le premier interrogatoire par le juge président, le mineur est examiné et observé par le médecin de l'établissement dans le poste anthropométrique. Les observations recueillies au cours des activités scolaires et pédagogiques complétent celles du médecin ${ }^{48}$. Parallèlement, une enquête de milieu pouvait être déclenchée auprès de la famille, des voisins ou l'entourage du mineur (patron, instituteur, etc.). Elle était confiée aux délégués de surveillance dont le modèle généralement évoqué est celui des probation officers américains, ce qui ne correspond pas tout à fait au rôle, fluctuant et imprécis, des débuts. Ils connaissent par ailleurs beaucoup de difficultés à démarrer, dû au fait de leur recrutement sur la base du volontariat, une pratique qui n'a pas de tradition au Portugal, sans parler de la méfiance de l'environnement à collaborer avec eux à cause
47. La création en 1930 de l'Institut Navarro de Paiva, malgré son ouverture tardive, est la première réponse conséquente à ce problème.

48. Relatorio do juiz presidente da Tutoria central da infância de Lisboa - Protecção de menores. Ano judicial de 1910-1911, Lisbonne, s. d. 
49. Oliveira Augusto de, «Relatorio dos serviços de menores delinquents »,

Boletim do Instituto de Criminologia, vol. III, $2^{\mathrm{e}}$ semestre, 1922, p. 145.

50. Santos João Miguel R. S., «Regime juridico... », p. 192. Basée sur l'évolution de la justice des mineurs, Gersão critique, comme contrepoint de cette flexibilité, «les pouvoirs discrétionnaires des différents intervenants " auprès des enfants de justice. Cela aurait pour conséquence de contribuer à ce que la " sélection » de ceux qui vont être internés « se limite, en fait, aux enfants des familles les plus pauvres et désorganisées, indépendamment du comportement criminel qu'elles révèlent ».

«Menores agentes...", p. 657-658. de leur image policière. La LPE parle même à ce propos de " corps de police spécialisé ». Avec le décret 10767, les procédures d'observation et d'examen vont se complexifier et s'élargir au-delà de la phase qui précède le jugement des mineurs délinquants. D'après ce décret, la solution du problème de la délinquance infantile reposait sur la soumission du mineur à un régime mixte qui comportait deux versants. Un versant juridique, correspondant aux mesures disciplinaires et punitives selon les différents types de délinquants. Et un versant médico-pédagogique, orienté vers la régénération du mineur, " qui ne peut plus être obtenue par les moyens applicables aux normaux mais par des moyens spécialisés de nature médico-pédagogique ». Et où l'enseignant et le médecin ont un rôle particulier à jouer en tant qu'experts principalement. Augusto de Oliveira insiste, à propos du médecin, sur l'importance de se restreindre à ce rôle d'" expert observateur, dans le laboratoire de psychologie infantile qu'est le refuge ainsi qu'au sein des établissements de réforme et de correction ${ }^{49}$ ". On prévoit l'existence dans chaque refuge d'un poste d'observation et d'examen anthropologique, médical et pédagogique, élargi à l'étude des tendances professionnelles et conditions socio-juridiques des mineurs et de leurs parents. Ces éléments sont enregistrés dans un bulletin biographique (le modèle de ce bulletin et de l'enquête sociale seront formalisés au cours de l'année 1925) en vue de son identification et de l'instruction du procès. Au-delà de cette phase, le décret stipule le contrôle judiciaire permanent basé sur les principes de l'individualisation et de l'indétermination. Aux examens et enquêtes effectués pendant le séjour du mineur au refuge, viennent s’ajouter par la suite de nouveaux examens et de nouvelles observations afin de déterminer les moyens et les méthodes de correction, les plus adaptés à chacun. Ce qui donne tout son sens à l'affirmation de Beleza dos Santos qui a cumulé pendant plusieurs années les fonctions de professeur de droit pénal avec celles de président du tribunal central pour enfants de Coimbra. Il caractérise le droit pénal des mineurs au Portugal comme « essentiellement subjectif, cherchant à adopter des mesures adaptables le plus possible à l'individualité du mineur, librement choisies par le tribunal et modifiables pendant leur exécution. Bref, flexibles, individualisées et modifiables ${ }^{50} "$.

Cette orientation traverse l'action en matière d'internement matérialisée dans la création par le décret 10767 des deux types d'établissements pour mineurs délinquants: les maisons de réforme (reformatorios) et les colonies 
correctionnelles (colonias correccionais). Nous ne pourrons pas développer ici ce point relatif au versant institutionnel de la justice des mineurs qui mérite à lui seul un long article. Nous nous limiterons donc à rappeler les critères qui président à l'application de la mesure. Les maisons de réforme sont destinées aux mineurs pas encore gravement pervertis et susceptibles d'être corrigés, pendant que les colonies correctionnelles s'adressent à ceux qui sont déjà gravement pervertis mais susceptibles encore d'être régénérés. Cela signifie que la législation portugaise ne reconnaît pas de mineurs incorrigibles a priori. Dans les deux types d'établissements, les moyens de base sont le travail professionnel, l'éducation morale et l'action disciplinaire, plus sévères dans le cas des colonies correctionnelles, accompagnés ou non de détention dans l'établissement d'une maximale de 60 jours, suivis ou alternés. À cette forme de détention (disciplinaire), s'ajoute, dans le cas des colonies correctionnelles, une autre, appelée correctionnelle, aussi dans l'établissement, de 60 jours à 5 ans, par des périodes variables et indéterminées, le temps d'internement global dans chacun des deux types d'établissements étant de 6 ans. Enfin, chaque mineur peut voir son régime d'internement atténué ou aggravé, sur la base de la procédure d'évaluation destinée à baliser son parcours de régénération, à travers le transfert de la colonie correctionnelle vers une maison de réforme ou vice-versa. C'est l'amorce du régime progressif qui est établi deux ans plus tard. Les travaux de Eliana Gersão et de Antonio Carlos Duarte-Fonseca ${ }^{51}$ nous amènent à penser que c'est sans doute là, sur le plan de l'internement, que le bilan de la justice des mineurs au Portugal est, malgré quelques expérience réussies, le moins brillant et l'écart entre les principes et la réalité, le plus frappant. Et ceci, à plusieurs titres: le recours quasi systématique à cette mesure par les tribunaux, critiqué d'ailleurs par Beleza dos Santos, dès 1925; la tendance à l'endurcissement du régime interne des établissements de réforme et de correction, etc. Mais ce fonctionnement s'est-il déroulé de façon uniforme, continue, au fil du temps? Voilà l'une des questions à laquelle seule une analyse approfondie des sources (primaires) permettra de répondre.

Quelles que soient ses limites (et nous en avons pointé quelques-unes) et quelle que puisse être la façon dont elle a été amenée à évoluer, la loi fondatrice de la justice des mineurs au Portugal ne cesse de surprendre. Par l'audace qu'a constituée, en 1911, l'option en faveur de la protection des mineurs, au moment où peu de pays en Europe encore, et dans le monde, avaient mis en
51. Duarte-Fonseca Antonio Carlos, Internamento de menores delinquentes, Coimbra, Coimbra Editora, 2005. Ce travail, issu d'un mémoire universitaire, bénéficie de l'expérience de l'auteur comme ancien directeur d'un établissement pour mineurs de justice. 
52. Gersao Eliana "Menores agentes... ", op. cit., p. 264-265.

place une telle juridiction. Mais aussi par l'innovation que représente, comme l'observe Eliana Gersão, le refus assumé des peines de prison pour les mineurs de moins de 16 ans.

Sur ce point, écrit-elle, notre régime est, encore aujourd'hui, plus "géné《reux" que la majorité des systèmes européens, où l'on accepte, généralement, la prison comme une possibilité, à partir d'une limite d'âge inférieure à celle adoptée entre nous, qui est de 16 ans ${ }^{52}$." 